\title{
Metal-Graphene Interaction Studied via Atomic Resolution Scanning Transmission Electron Microscopy
}

\author{
Recep Zan, ${ }^{+, \neq}$Ursel Bangert, ${ }^{*, \neq}$ Quentin Ramasse, ${ }^{\S}$ and Konstantin S. Novoselov ${ }^{\dagger}$ \\ ${ }^{\dagger}$ School of Physics and Astronomy and ${ }^{\ddagger}$ School of Materials, The University of Manchester, Manchester, M13 9PL, United Kingdom \\ ${ }^{\S}$ Laboratories, CCLRC Daresbury Laboratory, Warrington, WA4 4AD, United Kingdom \\ S Supporting Information
}

\begin{abstract}
Distributions and atomic sites of transition metals and gold on suspended graphene were investigated via high-resolution scanning transmission electron microscopy, especially using atomic resolution high angle dark field imaging. All metals, albeit as singular atoms or atom aggregates, reside in the omni-present hydrocarbon surface contamination; they do not form continuous films, but clusters or nanocrystals. No interaction was found between $\mathrm{Au}$ atoms and clean single-layer graphene surfaces, i.e., no $\mathrm{Au}$ atoms are retained on such surfaces. $\mathrm{Au}$ and also $\mathrm{Fe}$ atoms do, however, bond to clean few-layer graphene surfaces, where they assume $\mathrm{T}$ and $\mathrm{B}$ sites, respectively. $\mathrm{Cr}$ atoms were found to interact more strongly with clean monolayer graphene, they are possibly incorporated at graphene lattice imperfections and have been observed to catalyze dissociation of $\mathrm{C}-\mathrm{C}$ bonds. This behavior might explain the observed high frequency of $\mathrm{Cr}$-cluster nucleation, and the usefulness as wetting layer, for depositing electrical contacts on graphene.
\end{abstract}
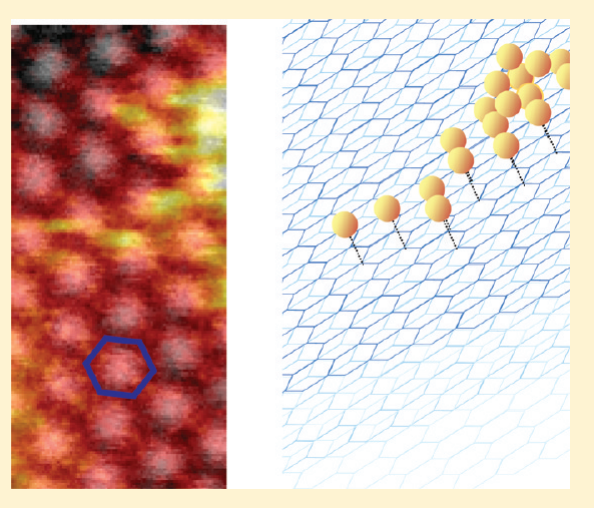

KEYWORDS: Graphene, STEM, gold, chromium, iron

\begin{abstract}
raphene, just one atom layer thick, has spurred a flurry of Iinvestigations into its structural properties, morphology, and chemistry. ${ }^{1,2}$ One of the fields of study concerns the interaction between metal and graphene. Our knowledge about this interaction to date is mainly based on theoretical calculations, many of these based on density functional theory (DFT). However, experimental exploration of the metal-graphene systems is still limited, and transmission electron microscopy (TEM) observations are practically nonexistent. Such observation would be of great interest to prove predicted positions of metal atoms in the hexagonal graphene structure. This is of importance not least in gaining an understanding of, e.g., the influence of metal contacts on macroscopic electrical transport.

Positions of foreign atoms on graphene have been calculated for a wide range of metals. $\mathrm{H}$ sites (center of the hexagon) are predicted mainly for alkaline metals, such as $\mathrm{K}, \mathrm{Na}, \mathrm{Cs}$, and $\mathrm{Ti}$, $\mathrm{Fe}$; $\mathrm{T}$ sites (top of carbon atom) for $\mathrm{Au}, \mathrm{Cu}, \mathrm{Ni}, \mathrm{Sn}$, and $\mathrm{F}$, whereas $\mathrm{Pt}, \mathrm{Cr}, \mathrm{Cl}, \mathrm{S}, \mathrm{O}, \mathrm{N}$, and $\mathrm{P}$ are expected to bind strongly to $\mathrm{B}$ sites, i.e., on top of a carbon-carbon bond (bridge site) as

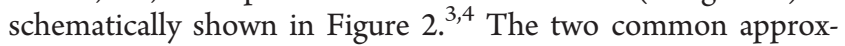
imations for DFT calculations, local density approximation and generalized gradient approximation, the latter does not represent van der Waals forces very well ${ }^{5}$, lead to different binding energies and thereby to different sites for Au atoms, $\mathrm{T}$ sites for the former and $B$ sites for the latter method. ${ }^{6}$ In addition, arbitrary variables used in the calculation, such as cutoff energy ${ }^{7}$ and size of the supercell ${ }^{8}$ can affect the result of DFT calculations. It was furthermore found, theoretically and experimentally, that point
\end{abstract}

defects (carbon vacancies) in graphene provide nucleation sites and even further substitutional incorporation of metals. ${ }^{4,9-13}$

Contrarily to high-resolution phase contrast imaging (HREM) in a transmission electron microscope, due to the approximate $\mathrm{Z}^{2}$-sensitivity dependency of high angle annular dark field (HAADF) image contrast, ${ }^{14}$ sites of singular metal atoms on graphene can directly be revealed. Because of the extremely bright $(\sim 100 \mathrm{pA})$, highly focused $(1 \AA)$ electron probe, dedicated scanning transmission electron microscopes with cold field emission guns and probe corrector (AC STEMs) are prime tools to achieve this, but there are very few of these in existence worldwide and HAADF studies of graphene are practically nonexistent. Here we present a study of atomic sites of metal atoms on suspended, single, and few-layer graphene, primarily using atomic resolution HAADF imaging in an AC STEM.

Two different techniques for the preparation of suspended graphene membranes were used, micromechanical cleavage and lift-off of chemical vapor deposition (CVD) films grown on $\mathrm{Ni}$ and $\mathrm{Cu}$ substrates. ${ }^{15^{-17}} \mathrm{Au}$ and $\mathrm{Cr}$ atoms were deliberately introduced by either thermal or e-beam evaporation at thicknesses of 0.2 and $5 \AA$. Fe atoms have been identified as residual impurities, presumably originating from acid treatment (ferric chloride).

Received: November 12, 2010

Revised: December 24, 2010

Published: January 27, 2011 

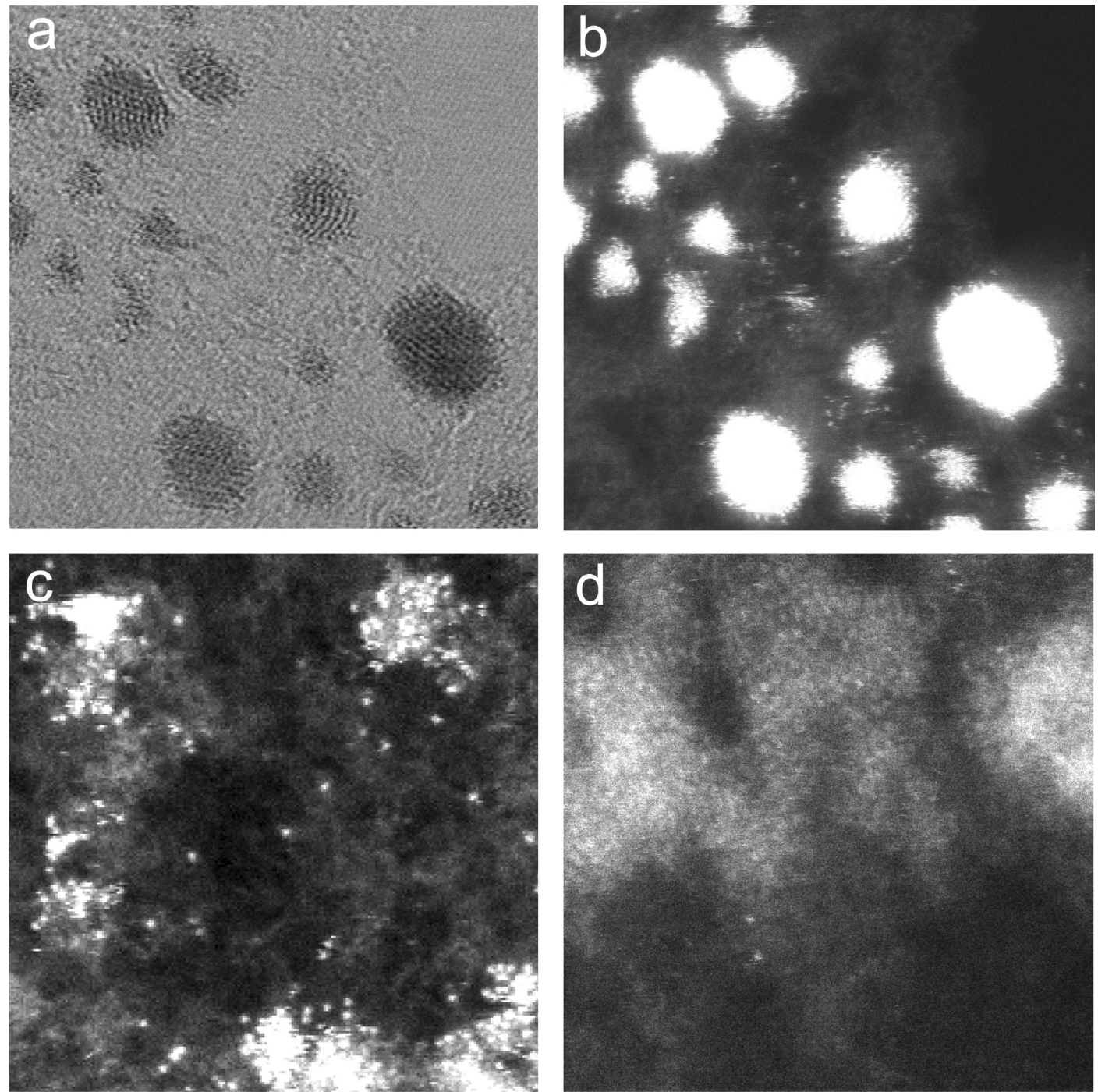

Figure 1. (a) BF and (b) HAADF image of monolayer graphene regions with $0.2 \mathrm{~A}$ of Au evaporated on top. Au nanocrystals are clearly visible in both images; the HAADF image furthermore reveals single Au atoms. Hydrocarbon contamination is manifest as wormlike background in the BF and as darkgray cloudlike contrast in the HAADF image. (c) HAADF image of $\mathrm{Fe}$ atoms on monolayer graphene. Note again the hydrocarbon deposit, which hosts the atoms. (d) HAADF image of a monolayer graphene region with $0.2 \AA \mathrm{Cr}$ evaporated on top. $\mathrm{Cr}$ atoms are spread over wide areas in noncrystalline agglomerates predominantly amidst hydrocarbon deposits. The frame width in all images is $10 \mathrm{~nm}$.

Additionally to identifying the graphene layer number via the plasmon energy ${ }^{18}$ we have simulated HAADF and bright field (BF) STEM images of pristine mono-, bi-, tri-, up to eight-layer graphene using Kirkland's HREM simulation software. ${ }^{19}$ Further to HAADF analysis all atom species were identified by ultrahigh spatially resolved electron energy loss spectroscopy (EELS).

Metal atom deposition can be affected by many factors, such as the substrate, ${ }^{20,21}$ which the graphene is placed upon, the temperature during the deposition, and the deposition rate, all resulting in different surface morphologies ${ }^{22}$ and transport properties. ${ }^{23}$ Extensive HAADF investigations in combination with electron energy loss spectroscopy (EELS), ${ }^{24}$ in which we have scrutinized many clean single-layer graphene patches for foreign atoms, never revealed impurity atoms with $Z \geq 9$ attached to single layer graphene sheets. However, we observed many types of impurities within the hydrocarbon contamination on single and multiple sheets, and we did also observe impurity atoms on clean areas in multilayer graphene. It was found previously that an energetically favorable path for all metal atoms is to form clusters, ${ }^{11}$ either at contamination sites and defects (in the case of single layers) or directly on the clean graphene surface of few-layer membranes.

First-principle calculations of gold atoms and dimers on graphene surfaces show that the gold-gold interaction is significantly stronger than the gold-graphene interaction ${ }^{6}$ (for this reason gold is highly mobile on graphene), as is experimentally confirmed by Gan et al. ${ }^{10}$ with observation of gold clustersrather than atoms - on the graphene sheet. For a single gold atom the favorable energy configuration is found to be directly above a carbon atom on a graphene sheet. An interesting result for the Au doping mechanism from DFT calculations, proposed by Pinto et al., ${ }^{25}$ is that Au does not dope single layers; however, it dopes bilayer graphene. We note that the following images are representative of a vast number of images obtained from many samples. We especially want to emphasize that the graphene surface seen as background in Figure 1a has a similar appearance in all graphene samples ever obtained by us, albeit via micromechanical exfoliation, from CVD grown samples, after cleaning 

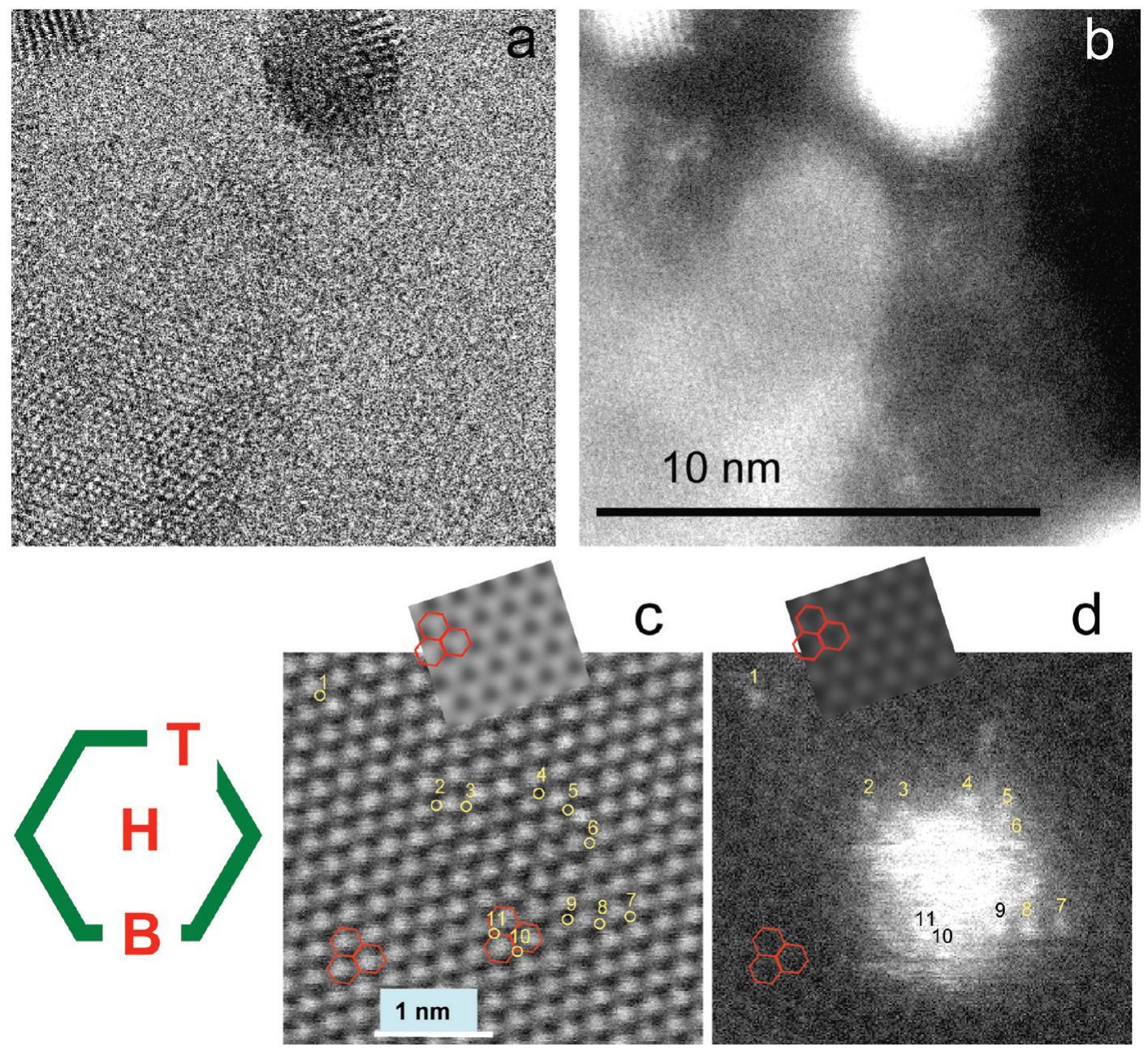

Figure 2. (a) BF STEM image of 0.2 Å gold evaporated on graphene, showing a monolayer gold-atom raft in the left bottom corner and a gold nanocrystal at the top. (b) Corresponding HAADF image. (c) Atomic resolution BF and (d) corresponding HAADF STEM image depicting a few-layer patch in graphene evaporated with 5 Å of gold. In (d) individual gold atoms can bee seen separated from the small cluster in the middle. Benzene rings are overlaid in red in both images, showing that bright contrast in the BF image corresponds to dark contrast in the centers of the top-layer benzene rings in the HAADF image, as derived from simulations of three-layer graphene (at $-50 \AA$ defocus), shown in the insets in (c) and (d). Single atoms are marked with numbers 1-11 just above the atoms in the HAADF image; identical places are marked by yellow circles in the BF image, identifying them to be $\mathrm{T}$ sites. The images represent raw, unfiltered data. Shown on the left of (c) is a schematic with metal sites on the benzene ring.

in solvents, or after plasma treatment: nanometer-size pristine graphene patches, constituting overall less than $\sim 30 \%$ of the total surface, are surrounded by hydrocarbon-based deposits. The latter are more or less mobile under the electron beam depending on their thickness.

We found that gold atoms form nanocrystalline clusters on graphene as shown in Figure 1a, and the gold cluster size increases with increasing evaporated Au. These clusters form exclusively on hydrocarbon contamination, revealed by grayish contrast in HAADF images (Figure $1 \mathrm{~b}$ ) or structural irregularities, e.g., defect sites, but never on clean graphene; we have never observed singular $\mathrm{Au}$ atoms. This demonstrates the extreme mobility of gold on pure graphene, suggesting that the interaction between graphene and gold is weak, similar to observations by Charlier et al. of Au-doped carbon nanotubes. ${ }^{9}$

Figure $2 \mathrm{a}$ shows an area in atomic resolution, where a monolayer of gold has formed on graphene (bottom left corner). The HAADF image in Figure 2b, by the dark grayish surround of this gold-atom raft, reveals that even this monolayer sits amidst hydrocarbon contamination. We did, however, observe individual
$\mathrm{Au}$ atoms on clean few-layer graphene patches. It thus appears that atoms in subsurface layers are needed to contribute to the bonding with surface gold atoms in order to prohibit dissociation and diffusion. Figure $2 c$ is a high-resolution BF STEM phase contrast image of few-layer graphene. The electron beam is focused on the exit surface of the sample, on which the gold is evaporated, and hence proceeds through the graphene layers before it encounters the gold atoms. The aggregate of gold atoms, seen in the HAADF image in Figure $2 \mathrm{~d}$ is so thin that it is invisible and not interpretable in phase contrast. However, the BF image exhibits strong contrast and relatively little noise and shows the lattice periodicities well, so it can be used as a guide to identify positions in the corresponding, simultaneously acquired HAADF image (Figure 2d), which has inherently much lower intensity. Here the graphene atomic lattice is barely recognizable, but the gold atoms are clearly visible. They have formed a loose aggregate with single atoms in their vicinity, some of which have been numbered. In order to locate the benzene rings we have carried out BF- and HAADF-STEM image simulations for few-layer graphene 

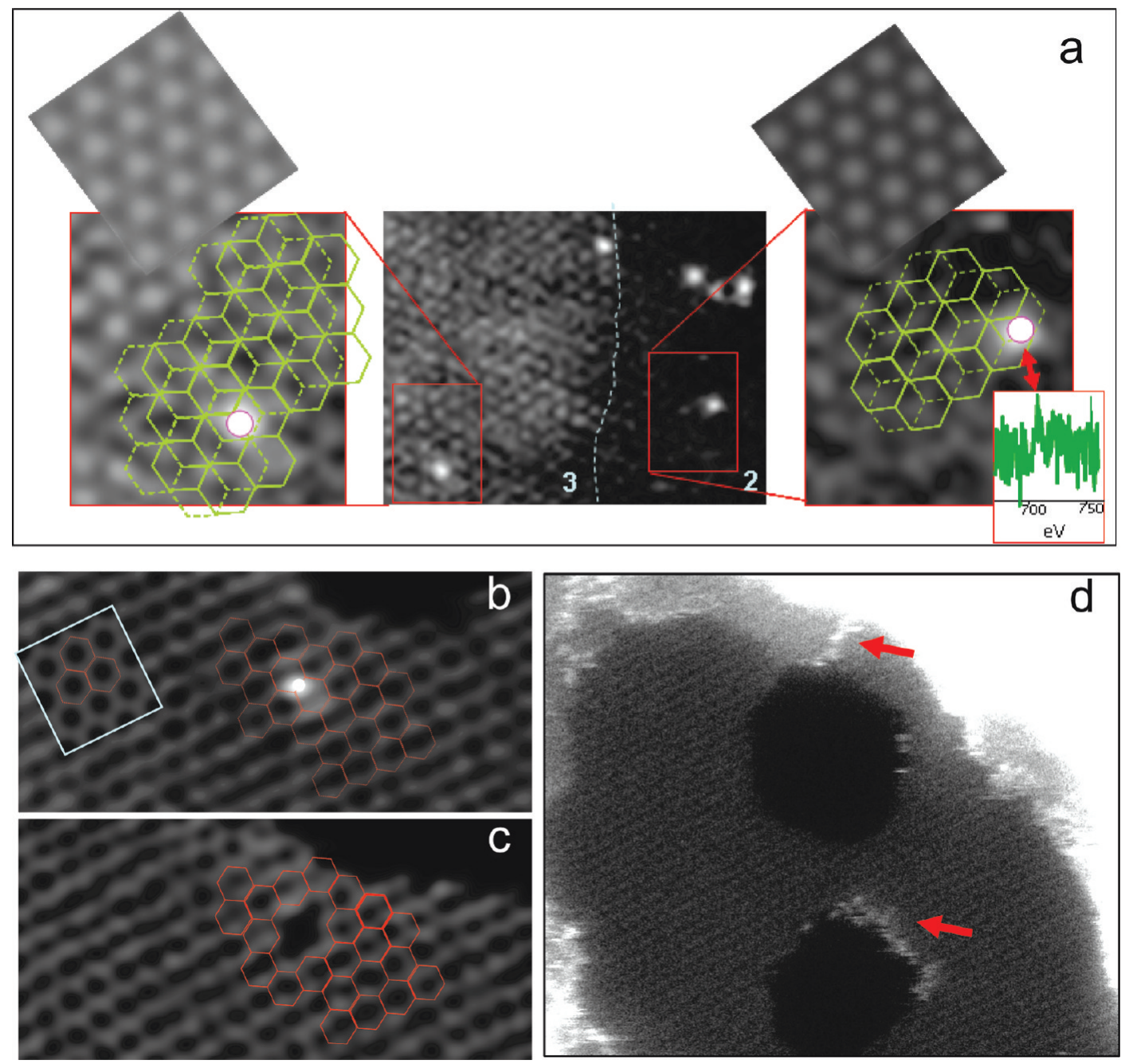

Figure 3. (a) Middle panel: Noise-reduced HAADF lattice image of two- and three-layer graphene with Fe impurities (the blue line shows the approximate position of the sheet edge). The left- and right-hand panels are enlarged views of the red frames, with overlaid model structures (solid lines indicate the surface layer) to clarify the position of the Fe atoms; these sit on the surface on B sites. Shown in the insets are HAADF simulations of three layers (left) and two layers (right). The spectrum inset shows the Fe L2,3 absorption edge obtained on the single, arrowed atom. (b) Noise-filtered HAADF image of a $\mathrm{Cr}$ atom on monolayer graphene; the HAADF image simulation is shown in the inset. (c) Same area as in (b) after repeat scanning, revealing a divacancy, where the $\mathrm{Cr}$ atom had been. Model structures are overlaid to show the sites of the defects. (d) Raw HAADF image of monolayer graphene patch (dark gray) bordered by hydrocarbons (lighter gray). Cr atoms sit on the hydrocarbon contamination; a chain of $\mathrm{Cr}$ atoms (arrowed; top) can be seen moving from an area of Cr clusters (white patch in the top right corner: the image is overexposed here due to the high $\mathrm{Cr}$ concentration) toward the edge of the top hole (black area) and to decorate the edge of the bottom hole (arrowed, bottom).

(with three layer simulations at $-50 \AA$ defocus giving the best match). These are shown as insets in panels $\mathrm{c}$ and $\mathrm{d}$ of Figure 2. We note that the benzene ring centers of the top layer correspond to bright patches in the BF image (c) and are dark in the HAADF image (d). The model structure (red benzene rings) is overlaid in identical locations on the simulations as well as on the experimental $\mathrm{BF}$ and HAADF image (in the bottom left corners). Hence $\mathrm{C}$ atoms-the hexagon corners-constitute bright contrast in HAADF images, and-under the current focusing conditions-dark contrast in BF images. On comparison of locations of gold atoms spotted in the HAADF image (circled) with identical positions in the BF image, the sites can be identified as $\mathrm{T}$ sites. This is in agreement with theoretical predictions.

Iron atoms have not been deliberately deposited in our study but reside as impurities on many samples, presumably as a result of chemical processing. The HAADF image in Figure 1c shows that, as in the previous case, on uncontaminated single layer areas (black patches) the sticking probability is very small: all $\mathrm{Fe}$ clusters and single atoms (white spots) are located on top of or at the edges of hydrocarbon contamination (grayish areas). On the surfaces of multilayer graphene on the other hand, individual Featoms can be seen. Figure 3a, shows a low-pass filtered atomic resolution HAADF image (noise reduced) of two and three staggered graphene sheets, using Fourier filtering provided in the Gatan imaging software. Fe atoms are clearly visible as white spots.

The EEL spectrum in the inset is taken on an individual $\mathrm{Fe}$ atom, showing the characteristic $\mathrm{L}_{2,3}$ absorption edge of $\mathrm{Fe}$ at $\sim 708 \mathrm{eV}$. In the left- and right-hand panels of Figure 3 a the areas around $\mathrm{Fe}$ atoms on three-layer and two-layer graphene, respectively, are enlarged with a model of the $A B$-stacked graphene lattice overlaid, the exact position of which was confirmed from simulated HAADF images (shown as insets). It becomes clear 
that the Fe atoms in both cases sit on B sites, in contradiction to calculations, which predict $\mathrm{H}$ sites.

Chromium, similar to gold and iron, was found at large on hydrocarbon deposits (Figure 1d). Cr was evaporated to the same $0.2 \AA$ layer thickness as $\mathrm{Au}$; however, the fractional coverage was larger (Figure 1d). This indicates that the Cr clusters are flatter or less dense than the Au clusters (e.g., compare panels b and $\mathrm{d}$ of Figure 1). Notably there does not appear to be a high degree of crystallinity in the $\mathrm{Cr}$ deposits, suggesting that the $\mathrm{Cr}-$ in contrast to the $\mathrm{Au}$-deposits in molecular form, possibly partially as $\mathrm{Cr}$ oxide; indeed EEL spectral images have proven that high $\mathrm{Cr}$ levels coincide with oxygen signals. Interestingly with $\mathrm{Cr}$, singular atoms do appear in some images on monolayer graphene. These atoms stay in place over several image scans. Figure $3 \mathrm{~b}$ shows an HAADF image of a $\mathrm{Cr}$ atom on a clean monolayer graphene patch. By overlaying the structural model, we identify the $\mathrm{Cr}$ position as a B site, as predicted from DFT models. After several scans the $\mathrm{Cr}$ atom has disappeared, leaving two vacancies behind (Figure.3c). Hence $\mathrm{Cr}$ is more reactive with graphene than $\mathrm{Au}$. Considering the relative stability of the $\mathrm{Cr}$ atom and the double vacancy after its disappearance, we cannot exclude the possibility that the $\mathrm{Cr}$ atom, rather than assuming a B site, was indeed incorporated to substitute two $\mathrm{C}$ atoms in the graphene sheet. Figure $3 b$ shows the edge of a hole in the graphene layer on the top right. The hole has become larger after repeated scanning in Figure 3c. From observations of many image scans in different areas, we can derive a possible mechanism for the hole formation: $\mathrm{Cr}$ atoms are frequently seen to decorate the edges of graphene layers (see bottom hole in the HAADF image in Figure 3d). The figure furthermore shows that $\mathrm{Cr}$ atoms (dotted chain indicated by top arrow) migrate from larger clusters (white contrast in top right corner) to the edge of the (top) hole. When the $\mathrm{Cr}$ atoms dissociate during repeat scans, i.e., have vanished from the images, the holes have enlarged. This is strong indication for $\mathrm{Cr}$-mediated $\mathrm{C}-\mathrm{C}$ dissociation and vacancy formation.

We want to note here that in order to achieve improved "sticking" and incorporation of metals in graphene we also introduced atoms (e.g., cobalt) via low-energy ion implantation at $200 \mathrm{eV}$ to a density of typically 1 atom $\mathrm{nm}^{-2}$. The results were very similar to those of the Fe impurities on graphene with all observed Co sitting on hydrocarbons. It was, however, not possible to identify atomic Co sites within/on top of the graphene sheet; not one single Co atom could be detected in clean graphene areas (results not shown here).

In conclusion, investigation of transition metals and gold on graphene showed that clean single-layer graphene surfaces do not retain any significant amount of atoms of these species (notably none in the case of gold). All metals, albeit as singular atoms or clusters thereof reside in the abundant hydrocarbon surface contamination. This behavior might have consequences for macroscopic electrical transport properties in graphene. Although electrical transport measurements are usually obtained from graphene supported by substrates, the graphene membrane even in this case is still a 2-D object. Because contamination appears to be an issue with 2-D structures, it is very likely that charge transfer from monolayer graphene into metal contacts proceeds in the largest part across a macromolecular layer (hydrocarbons). Individual $\mathrm{Au}$ and $\mathrm{Fe}$ atoms could be observed, however, on clean few-layer graphene surfaces and found to reside on top of carbon atoms and on top of $\mathrm{C}-\mathrm{C}$ bonds, respectively. Chromium appears to bond more strongly to monolayer graphene and has been observed to catalyze dissociation of $\mathrm{C}-\mathrm{C}$ bonds. Bonding of singular $\mathrm{Cr}$ atoms to graphene, possibly via lattice defects, could be a precursor for the highfrequency nucleation of $\mathrm{Cr}$ clusters, which, in contrast to $\mathrm{Au}$, provide more continuous contact throughout the membrane, and hence the importance of the role of a $\mathrm{Cr}$ "wetting layer" between $\mathrm{Au}$ and graphene in forming electrical contacts on graphene, which is commonly used in electrical transport studies, can be understood.

\section{ASSOCIATED CONTENT}

S Supporting Information. Descriptions of sample preparation, EM tools and evaluations, and image simulation. This material is available free of charge via the Internet at http://pubs.acs.org.

\section{AUTHOR INFORMATION}

\section{Corresponding Author}

*E-mail: bangert.ursel@manchester.ac.uk.

\section{REFERENCES}

(1) Novoselov, K. S.; Geim, A. K.; Morozov, S. V.; Jiang, D.; Zhang, Y.; Dubonos, S. V.; Grigorieva, I. V.; Firsov, A. A. Science 2004, 306 (5696), 666-669.

(2) Geim, A. K.; Novoselov, K. S. Nat. Mater. 2007, 6 (3), 183-191.

(3) Chan, K. T.; Neaton, J. B.; Cohen, M. L. Phys. Rev. B 2008, 77 (23), No. 235430.

(4) Boukhvalov, D. W.; Katsnelson, M. I. Appl. Phys. Lett. 2009, 95 (2), No. 023109.

(5) Leenaerts, O.; Partoens, B.; Peeters, F. M. Phys. Rev. B 2008, 77 (12), No. 125416.

(6) Akturk, U.; Olcay; Tomak, M. Phys. Rev. B 2009, 80 (8), No. 085417.

(7) Varns, R.; Strange, P. J. Phys.: Condens. Matter 2008, 20 (22), No. 225005.

(8) Leenaerts, O.; Partoens, B.; Peeters, F. M. Microelectron. J. 2009, 40 (4-5), 860-862.

(9) Charlier, J. C.; et al. Nanotechnology 2009, 20 (37), No. 375501.

(10) Gan, Y.; Sun, L.; Banhart, F. Small 2008, 4 (5), 587-591.

(11) Krasheninnikov, A. V.; Lehtinen, P. O.; Foster, A. S.; Pyykkö; Nieminen, R. M. Phys. Rev. Lett. 2009, 102 (12), No. 126807.

(12) Zhou, Y. G.; Zu, X. T.; Gao, F.; Lv, H. F.; Xiao, H. Y. Appl. Phys. Lett. 2009, 95 (12), No. 123119.

(13) Okamoto, Y. Chem. Phys. Lett. 2006, 420 (4-6), 382-386.

(14) Wang, Z. L.; Cowley, J. M. Ultramicroscopy 1989, 31 (4), 437453.

(15) Li, X.; Cai, W.; An, J.; Kim, S.; Nah, J.; Yang, D.; Piner, R.; Velamakanni, A.; Jung, I.; Tutuc, E.; Banerjee, S. K.; Colombo, L.; Ruoff, R. S. Science 2009, 324 (5932), 1312-1314.

(16) Kim, K. S.; Zhao, Y.; Jang, H.; Lee, S. Y.; Kim, J. M.; Kim, K. S.; Ahn, J.-H.; Kim, P.; Choi, J.-Y.; Hong, B. H. Nature 2009, 457 (7230), 706-710.

(17) Reina, A.; Jia, X.; Ho, J.; Nezich, D.; Son, H.; Bulovic, V.; Dresselhaus, M. S.; Kong, J. Nano Lett. 2008, 9 (1), 30-35.

(18) Eberlein, T.; Bangert, U.; Nair, R. R.; Jones, R.; Gass, M.; Bleloch, A. L.; Novoselov, K. S.; Geim, A.; Briddon, P. R. Phys. Rev. B 2008, 77 (23), No. 233406.

(19) Kirkland, E. J. Advanced Computing in Electron Microscopy, 1st ed.; Plenum Press: New York, 1998.

(20) Zhou, Z.; Gao, F.; Goodman, D. W. Surf. Sci. 2010, 604 (13-14), L31-L38.

(21) Alpha, T. N. D.; et al. New J. Phys. 2009, 11 (10), No. 103045.

(22) Sicot, M.; Bouvron, S.; Zander, O.; Rudiger, U.; Dedkov, Y. S.; Fonin, M. Appl. Phys. Lett. 2010, 96 (9), No. 093115. 
(23) McCreary, K. M.; Pi, K.; Swartz, A. G.; Han, W.; Bao, W.; Lau, C. N.; Guinea, F.; Katsnelson, M. I.; Kawakami, R. K. Phys. Rev. B 2010, 81 (11), No. 115453.

(24) Bangert, U.; Bleloch, A.; Gass, M. H.; Seepujak, A.; van den Berg, J. Phys. Rev. B 2010, 81 (24), No. 245423.

(25) Pinto, H.; Jones, R.; Goss, J. P.; Briddon, P. R. Phys. Status Solidi A 2010, 207 (9), 2131-2136. 